\title{
Lensing Studies with Diffuse Backgrounds
}

\author{
Asantha Cooray \\ Theoretical Astrophysics, California Institute of Technology, Pasadena, California \\ 91125
}

\begin{abstract}
The current weak lensing measurements of the large scale structure are mostly related to statistical study of background galaxy ellipticities. We consider a possibility to extend lensing studies with intrinsically unresolved sources and suggest that spatial fluctuations in the integrated diffuse emission from these sources can be used for a lensing reconstruction. Examples of upcoming possibilities include the diffuse background generated by dusty starburst galaxies at far-infrared wavelengths, first stars and galaxies in near-infrared wavelengths, and the background related to $21 \mathrm{~cm}$ emission by neutral gas in the general intergalactic medium prior to reionization. While methods developed to extract lensing information from cosmic microwave background (CMB) temperature and polarization data can be easily modified to study lensing properties using diffuse backgrounds at other wavelengths, we suggest that the lensing extraction from these backgrounds using higher order non-Gaussian clustering information alone may not be the best approach. In contrast to CMB anisotropies, reasons for this include the lack of features in the clustering power spectrum such that the resulting lensing modification to the angular power spectrum of low-redshift diffuse backgrounds, at arcminute angular scales, is insignificant. While the use of low redshift backgrounds for lensing studies will be challenging, due to confusing foregrounds among other reasons, the use of suggested backgrounds will extend the reconstruction of the integrated matter power spectrum out to redshifts of 15 to 30, and will bridge the gap between current and upcoming galaxy lensing studies out to, at most, a redshift of a few and planned weak lensing studies with CMB out to the last scattering surface at a redshift of 1100 .
\end{abstract}

\section{Introduction}

The weak gravitational lensing of background galaxies by the intervening large scale structure is now a well known probe of physical cosmology (Kaiser 1992; Kaiser 1998; Jain \& Seljak 1997; Hu \& Tegmark 1999; Bartelmann \& Schneider 2001). The current weak lensing studies are mostly limited to reduced-shear

Preprint submitted to Elsevier Science 29 October 2018 
estimates that involve statistical study of galaxy ellipticities or shape information reconstructed through expansions related to certain orthogonal basis functions (for a recent review, see, Refregier 2003). Such a lensing reconstruction, unfortunately, is limited to resolved background sources for which reliable shape measurements, after accounting for the point spread function, are possible. While statistics of current and most upcoming weak lensing surveys are expected to be limited by instrumental constraints, such as issues related to the point spread function, with reasonable improvements on the observational side, the statistics related to large scale structure lensing studies will eventually be limited by the number of resolved background sources. In this limit, to further improve mass reconstruction related to lensing, background objects which are intrinsically point-sources, and, thus, for which shape measurements are impossible, must be included in the analysis. A situation where point-sources dominate resolved source number counts is already present in some of the deepest space-based images available to date, including North and South Hubble Deep Fields and recent images from the Advanced Camera for Surveys (ACS) on the Hubble Space Telescope.

As one moves to higher source redshifts, beyond what is currently encountered in large scale structure lensing studies, the importance of point sources is more evident; The first stars and proto galaxies, at redshifts of order 15 to 30 and higher, provide an ideal background for lensing studies given the large path length to these background sources. While the detection of an individual first star is beyond the capabilities of even next generation instruments, the spatial fluctuations in the integrated diffuse background emission from these stars, at least in most optimistic models of their emission, may be within reach (e.g., Cooray et al. 2003). During the transit to us, this diffuse emission is expected to be affected by the intervening mass distribution via gravitational lensing and provides a background for a potentially interesting lensing study.

Contrary to background intensities at optical and infra-red wavelengths (Cooray et al. 2003; Knox et al. 2001), where the emission can eventually be broken down to point sources, one also encounter backgrounds which are composed of truly diffuse emission that cannot easily be separated to individual objects. Examples of such diffuse backgrounds are the $21 \mathrm{~cm}$ rest wavelength emission from neutral Hydrogen in the intergalactic medium prior to reionization (Scott \& Rees 1990; Madau et al. 1997; Tozzi et al. 1997; Iliev et al. 2002; Furlanetto et al. 2003) and, of course, the cosmic microwave background (CMB) radiation. The lensing effect on CMB temperature and polarization fluctuations, that were generated at the last scattering surface (Peebles \& Yu 1970; Sunyaev \& Zel'dovich 1970; Silk 1968; Hu \& Dodelson 2001), is now well understood (Seljak 1996; Metcalf \& Silk 1997; Hu 2000) and various techniques have been developed to extract lensing information from high resolution CMB temperature and polarization maps (Zaldarriaga \& Seljak 1999; Benabed et al. 2001; Guzik et al. 2000; Hu 2001; Hu \& Okamoto 2002; Cooray \& Kesden 2003; 
Hirata \& Seljak 2003). These techniques make use of the fact that at the last scattering surface, where primary CMB temperature and polarization fluctuations are generated, these anisotropies follow a Gaussian distribution, while with gravitational lensing deflections when propagating to us, the fluctuations pattern on the sky is non-Gaussian (Bernardeau 1997; Hu 2001; Zaldarriaga 2000; kesden et al. 2002). The lensing extraction techniques make use of measurements related to certain non-Gaussian aspects, such as a collapsed form of the trispectrum (Zaldarriaga 2000; Hu 2001), which is related to a filtered version of the squared-temperature power spectrum (Cooray \& Kesden 2003), to directly estimate and reconstruct the deflection angle or the projected lensing potential power spectrum.

In the case of CMB, the study of lensing and its extraction is usually considered under a perturbative approach with the deflection angle as a small change to the anisotropy on the sky towards a given direction (Hu 2000; Goldberg \& Spergel 1998; Cooray \& Hu 1998; Zaldarriaga 2000). Under such an approach, for most practical purposes, the lensing effect can be calculated up to the second order in the deflection angle or first order in the lensing potential power spectrum. Since the rms deflection angle for a photon propagating from a redshift of 1100 is of order a few arcminute, a resulting complication here is that perturbative description of the lensing effect breaks down at small angular scales at and below the rms deflection angle. This, however, is not a major concern for the CMB lensing description since there is no intrinsic small angular scale power at these same angular scales due to the presence of significant damping. On the other hand, when compared to CMB temperature fluctuations, diffuse backgrounds at lower redshifts are expected to show substantial clustering power at arcminute angular scales and the perturbative approach just to the first order in the deflection power spectrum may not be an adequate description. Another important difference, when compared to CMB anisotropies that were generated at the last scattering surface with narrow range in redshift, is that background sources may be distributed over a wide range in redshift and this distribution must be properly accounted as well.

While there are complications from foregrounds and other confusions, we suggest that techniques developed to extract lensing information from CMB data can also be extended and applied at other wavelengths for lensing studies. In the case of $\mathrm{CMB}$ anisotropies, there are substantial modifications to the anisotropy angular power spectrum from the lensing effect due to the acoustic peak structure and the presence of a damping tail such that lensing transfers power from large scales to arcminute scales where the lensing effect is easily detectable. When compared to a lensing study with CMB, the lensing information one can extract from low redshift diffuse backgrounds is significantly limited. As we will discuss, this results from the fact that, at arcminute angular scales, the lensing modification to the power spectrum is minor and the non-Gaussianities generated are relatively smaller. This, again, is due to the 
lack of features in the clustering power spectrum such that the modification to the clustering pattern, at arcminute angular scales of interest for future observations, remains the same under gravitational lensing. The lack of significant differences between the intrinsic and lensed power spectra, thus, limits the use of statistics such as clustering variance for lensing purposes.

To extract lensing information better, one require arcsecond scale clustering information, though at such small angular scales, a substantial number of sources that contribute to the diffuse background will begin to be resolved. In the limit that most or all sources are resolved to point-like objects, instead of shear reconstruction based on source shapes, statistics related to the distribution of the point sources can also be used for a shear estimation. As an example, instead of extracting information from the non-Gaussian clustering information, one can make use of the anisotropy of clustering generated by foreground lensing. This anisotropy can be used as an estimator of shear and can be approached in the same manner ellipticity information from galaxy shapes are used for an estimate of shear. Such a study has already been attempted towards known regions of mass concentrations, such as galaxy clusters (van Waerbeke et al. 1997). Similar studies can be extended to large angular scales and the large scale structure as a whole when carefully selected backgrounds are selected for lensing reconstruction so as to minimize confusions resulting from foregrounds. Another aspect is the change resulting from lensing magnification and several suggestions have already been made to include unresolved sources in weak lensing studies through variations associated with number counts and, when measurable, source sizes (Jain 2002; Pen 2003).

The paper is organized as follows. In the next section, we discuss the lensing effect on diffuse backgrounds and extend calculations related to weak lensing effect on CMB anisotropies. As an example, we discuss the lensing modification of the near-IR anisotropy fluctuations due to first stars following calculations, related to spatial clustering discussed in Cooray et al. (2003). We discuss the extent to which lensing information can be extracted from this and other backgrounds and a comparison to the lensing extraction with CMB data. We also consider potential biases and systematic effects for a lensing reconstruction and conclude with a summary.

\section{Calculation}

\subsection{Lensing Effect on Clustering of Diffuse Backgrounds}

In order to derive the weak lensing effect on angular clustering properties

of any diffuse background, we follow a calculation similar to that introduced 
for the lensing extraction from CMB temperature anisotropies (e.g., Hu 2001; Seljak \& Zaldarriaga 1999; Cooray \& Kesden 2003) and make use of the flat sky approximation. When compared to CMB, there is one important difference in that one should properly account for the broad redshift distribution of sources that contribute to the background light, instead of the assumption related to a narrow source redshift distribution. Thus, we break the background intensity as

$$
I_{\mathrm{tot}}(\hat{\mathbf{n}})=\int_{0}^{\infty} d r \delta I(r \hat{\mathbf{n}}, r),
$$

where $\delta I(r \hat{\mathbf{n}}, r)$ is the fractional contribution to the total emission as a function of the radial distance or look-back time, from the observer:

$$
r(z)=\int_{0}^{z} \frac{d z^{\prime}}{H\left(z^{\prime}\right)},
$$

when the expansion rate for adiabatic CDM cosmological models with a cosmological constant is given by

$$
H^{2}=H_{0}^{2}\left[\Omega_{m}(1+z)^{3}+\Omega_{K}(1+z)^{2}+\Omega_{\Lambda}\right] .
$$

Here, $H_{0}$ can be written as the inverse Hubble distance today $c H_{0}^{-1}=2997.9 h^{-1}$ Mpc. We follow the conventions that in units of the critical density $3 H_{0}^{2} / 8 \pi G$, the contribution of each component is denoted $\Omega_{i}, i=c$ for the CDM, $b$ for the baryons, $\Lambda$ for the cosmological constant. We also define the auxiliary quantities $\Omega_{m}=\Omega_{c}+\Omega_{b}$ and $\Omega_{K}=1-\sum_{i} \Omega_{i}$, which represent the matter density and the contribution of spatial curvature to the expansion rate respectively. Note that, though we discuss a general derivation of lensing effect on background source clustering, we show results for the currently favorable $\Lambda$ CDM cosmology with $\Omega_{b}=0.05, \Omega_{m}=0.35, \Omega_{\Lambda}=0.65$ and $h=0.65$.

Weak lensing deflects the path of background photons resulting in a remapping of the observed anisotropy pattern on the sky, such that the fractional contribution from each redshift is modified as

$$
\begin{aligned}
& \tilde{\delta} I(r \hat{\mathbf{n}}, r)=\delta I[r\{\hat{\mathbf{n}}+\nabla \phi(r \hat{\mathbf{n}}, r)\}, r] \\
\approx & \delta I(r \hat{\mathbf{n}}, r)+\nabla_{i} \phi(r \hat{\mathbf{n}}, r) \nabla^{i} \delta I(r \hat{\mathbf{n}}, r)+\frac{1}{2} \nabla_{i} \phi(r \hat{\mathbf{n}}, r) \nabla_{j} \phi(r \hat{\mathbf{n}}, r) \nabla^{i} \nabla^{j} \delta I(r \hat{\mathbf{n}}, r)+\ldots(4)
\end{aligned}
$$

Here, $\delta I(r \hat{\mathbf{n}}, r)$ is the unlensed fractional component of the background light from a distance of $r, \tilde{\delta I}(r \hat{\mathbf{n}}, r)$ is the same contribution when affected by the gravitational lensing deflections during the transit, and $\nabla \phi(r \hat{\mathbf{n}}, r)$ represents 
the lensing deflection angle for a photon propagating from a distance of $r$. The second line of Eq. 4 treats the lensing deflection as a perturbative parameter. Typical lensing calculations related to CMB (e.g., Hu 2000), treat lensing effect on the angular power spectrum to the second order in this perturbation expansion, while lensing extraction techniques related to CMB consider information from the first order correction (e.g., Seljak \& Zaldarriaga 2000; Hu 2001). Such an approach works well since CMB has no intrinsic anisotropy at small angular scales below few arcminutes, though the typical deflection angle of a photon from the last scattering surface is $\sim 2.5$ arcminutes. The accuracy to which lensing can be extracted from CMB under this perturbative approach is discussed in Hirata \& Seljak (2003). Here, for low redshift diffuse backgrounds, the perturbation expansion up to second order in the deflection angle is not adequate to fully account for the lensing effect on the clustering power spectrum. We extend the calculation explicitly to higher order and present an exact derivation of the lensed power spectrum which should be valid even at small angular scales where the lensing deflection angle is of the same order. To properly evaluate the lensed contribution, however, one must evaluate an infinite series of integrals which is numerically exhaustive; here, in presenting numerical results, we only consider the lensed power spectrum up to the fourth order in deflection angle or the second order in the deflection-angle power spectrum.

In equation 4 , where $\nabla \phi$ is the deflection angle, $\phi$ is a radial projection of the gravitational potential, $\Phi$ (see, e.g. Kaiser 1992), for a source at a distance $r_{s}$

$$
\phi\left(r_{s} \hat{\mathbf{m}}, r_{s}\right)=-2 \int_{0}^{r_{s}} d r^{\prime} \frac{d_{A}\left(r_{s}-r^{\prime}\right)}{d_{A}\left(r_{s}\right) d_{A}\left(r^{\prime}\right)} \Phi\left(r^{\prime} \hat{\mathbf{m}}, r^{\prime}\right),
$$

where the comoving angular diameter distance is

$$
d_{A}=H_{0}^{-1} \Omega_{K}^{-1 / 2} \sinh \left(H_{0} \Omega_{K}^{1 / 2} r\right) .
$$

Note that as $\Omega_{K} \rightarrow 0, d_{A} \rightarrow r$.

Taking the Fourier transform, as appropriate for a flat-sky, we write the modification to the fraction contribution arising at a distance $r$ as

$$
\begin{aligned}
\tilde{\delta I}\left(\mathbf{l}_{1}\right) & =\int d \hat{\mathbf{n}} \tilde{\delta I}(\hat{\mathbf{n}}) e^{-i \mathbf{l}_{1} \cdot \hat{\mathbf{n}}} \\
& =\delta I\left(\mathbf{l}_{1}\right)-\int \frac{d^{2} \mathbf{l}_{1}^{\prime}}{(2 \pi)^{2}} \delta I\left(\mathbf{l}_{1}{ }^{\prime}\right) L\left(\mathbf{l}_{1}, \mathbf{l}_{1}{ }^{\prime}\right)
\end{aligned}
$$

where 


$$
\begin{aligned}
& L\left(\mathbf{l}_{1}, \mathbf{l}_{1}{ }^{\prime}\right) \equiv \phi\left(\mathbf{l}_{1}-\mathbf{l}_{1}{ }^{\prime}\right)\left(\mathbf{l}_{1}-\mathbf{l}_{1}{ }^{\prime}\right) \cdot \mathbf{l}_{1}{ }^{\prime}-\frac{1}{2} \int \frac{d^{2} \mathbf{l}_{1}^{\prime \prime}}{(2 \pi)^{2}} \phi\left(\mathbf{l}_{1}^{\prime \prime}\right) \\
\times & \phi\left(\mathbf{l}_{1}-\mathbf{l}_{1}{ }^{\prime}-\mathbf{l}_{1}{ }^{\prime \prime}\right)\left(\mathbf{l}_{1}^{\prime \prime} \cdot \mathbf{l}_{1}{ }^{\prime}\right)\left(\mathbf{l}_{1}-\mathbf{l}_{1}{ }^{\prime}-\mathbf{l}_{1}{ }^{\prime \prime}\right) \cdot \mathbf{l}_{1}{ }^{\prime}+\frac{1}{6} \int \frac{d^{2} \mathbf{l}_{1}^{\prime \prime}}{(2 \pi)^{2}} \int \frac{d^{2} \mathbf{l}_{1}^{\prime \prime \prime}}{(2 \pi)^{2}} \phi\left(\mathbf{l}_{1}{ }^{\prime \prime}\right) \phi\left(\mathbf{l}_{1}{ }^{\prime \prime \prime}\right) \\
\times & \phi\left(\mathbf{l}_{1}-\mathbf{l}_{1}{ }^{\prime}-\mathbf{l}_{1}{ }^{\prime \prime}-\mathbf{l}_{1}{ }^{\prime \prime \prime}\right)\left(\mathbf{l}_{1}{ }^{\prime \prime} \cdot \mathbf{l}_{1}{ }^{\prime}\right)\left(\mathbf{l}_{1}{ }^{\prime \prime \prime} \cdot \mathbf{l}_{1}{ }^{\prime}\right)\left(\mathbf{l}_{1}-\mathbf{l}_{1}{ }^{\prime}-\mathbf{l}_{1}{ }^{\prime \prime}-\mathbf{l}_{1}{ }^{\prime \prime \prime}\right) \cdot \mathbf{l}_{1}{ }^{\prime} \cdot+\ldots
\end{aligned}
$$

Here, for simplicity, we have dropped the explicit dependence on the radial distance, $r$, in $\delta I$ and $\phi$, and have expanded the correction to the third order in $\phi$.

We define the power spectrum of the total intensity fluctuation field, in the flat sky approximation, following the usual way

$$
\left\langle I(\mathbf{l}) I\left(\mathbf{l}^{\prime}\right)\right\rangle \equiv(2 \pi)^{2} \delta_{\mathrm{D}}\left(\mathbf{l}+\mathbf{l}^{\prime}\right) C_{l}
$$

where $\delta_{D}$ is the Dirac delta function. Similarly, the power spectrum of the lensed fluctuation field, with $I(\mathbf{l})$ replaced by $\tilde{I}(\mathbf{l})$, is $\tilde{C}_{l}$.

Note that in the absence of lensing, the observed anisotropy power spectrum is simply the sum of anisotropy contributions over the normalized source redshift distribution such that $C_{l}=\int d r C_{l}^{\delta I}$. The observed angular power spectrum, however, consists of both the unlensed intensity and a perturbative correction related to the lensing effect. To calculate the final lensed-clustering power spectrum, we first substitute Eq. 7 in Eq. 1 and reintroduce the dependence on distance. The lensing contribution to the power spectrum is calculated following approaches used to describe lensing effect on CMB anisotropies (e.g., $\mathrm{Hu} 2000$ ) and after some straight forward calculations, we obtain, to the fourth order in $\phi$ or second order in $C_{l}^{\phi}$ :

$$
\begin{aligned}
& \tilde{C}_{l}=C_{l}-l^{2} \int d r R(r) C_{l}^{\delta I}\left[1-\frac{l^{2}}{2} R(r)\right] \\
+ & \int \frac{d^{2} \mathbf{l}_{1}}{(2 \pi)^{2}} \int d r C_{l_{1}}^{\phi}(r) C_{\left|\mathbf{l}-\mathbf{l}_{1}\right|}^{\delta I}\left[\left(\mathbf{l}-\mathbf{l}_{1}\right) \cdot \mathbf{l}_{1}\right]^{2}\left[1-\left|\mathbf{l}-\mathbf{l}_{1}\right|^{2} R(r)\right] \\
+ & \frac{1}{2} \int \frac{d^{2} \mathbf{l}_{1}}{(2 \pi)^{2}} \int \frac{d^{2} \mathbf{l}_{2}}{(2 \pi)^{2}} \int d r C_{l_{1}}^{\phi}(r) C_{l_{2}}^{\phi}(r) C_{\left|\mathbf{l}-\mathbf{l}_{1}-\mathbf{l}_{2}\right|}^{\delta I}\left[\left(\mathbf{l}-\mathbf{l}_{1}-\mathbf{l}_{2}\right) \cdot \mathbf{l}_{1}\right]^{2}\left[\left(\mathbf{l}-\mathbf{l}_{1}-\mathbf{l}_{2}\right) \cdot \mathbf{l}_{2}\right]^{2},
\end{aligned}
$$

where $C_{l}^{\delta I}$ is the fractional contribution to the intensity fluctuation power spectrum as a function of the radial distance (see, Section 2.2) and

$$
R(r)=\frac{1}{4 \pi} \int d l l^{3} C_{l}^{\phi}(r)
$$


The cumulative variance of intensity fluctuations is conserved under lensing such that $\sigma^{2} \equiv \int d^{2} \mathbf{l} /(2 \pi)^{2} \tilde{C}_{l}=\int d^{2} \mathbf{l} /(2 \pi)^{2} C_{l}$; this is equivalent to the fact that lensing does not create or destroy power but rather results in a redistribution such that surface brightness remains conserved. Under lensing, however, the filtered variance of fluctuations as a function of angular scale is different such that $\sigma^{2}(\theta) \equiv \int d^{2} \mathbf{l} /(2 \pi)^{2} \tilde{C}_{l} W^{2}(l \theta)$, where $W(l \theta)$ is the Fourier transform of the filter - such as top-hat, Gaussian etc. - is different from that expected from the unlensed clustering power spectrum, $\int d^{2} \mathbf{l} /(2 \pi)^{2} C_{l} W^{2}(l \theta)$. While the modification to variance resulting from lensing can be used for a reconstruction of convergence (Pen 2003), such a measurement only works out accurately if the differences between $\tilde{C}_{l}$ and $C_{l}$ is significant, as a function of angular scale or multipole $l$, under gravitational lensing. While this is the case for arcminute scale power spectrum of CMB anisotropies, we will show that the difference between intrinsic- and lensed-power spectra of diffuse backgrounds is insignificant and will complicate a straight forward lensing reconstruction based on the variance alone.

In the case of CMB anisotropies, in Eq. $1, \delta I(r \hat{\mathbf{n}}, r)=I^{\mathrm{cmb}}(\hat{\mathbf{n}}) \delta_{D}\left(r-r_{0}\right)$ where $r_{0}$ is the distance to the surface of last scattering and $I^{\mathrm{cmb}}(\hat{\mathbf{n}})$ is the total $\mathrm{CMB}$ intensity such that for spatial fluctuations, $C_{l}^{\delta I}=C_{l}^{\mathrm{cmb}} \delta_{D}\left(r-r_{0}\right)$ with the primordial anisotropy power spectrum given by $C_{l}^{\mathrm{cmb}}$. This assumption simplifies Eq. 10 to a result valid for any background source of narrow width in distance or in redshift space, as

$$
\begin{aligned}
& \tilde{C}_{l}^{\mathrm{CMB}}=C_{l}^{\mathrm{CMB}}\left[1-l^{2} R\left(r_{0}\right)+\frac{l^{4}}{2} R^{2}\left(r_{0}\right)\right] \\
+ & \int \frac{d^{2} \mathbf{l}_{1}}{(2 \pi)^{2}} C_{l_{1}}^{\phi}\left(r_{0}\right) C_{\left|\mathbf{l}-\mathbf{l}_{1}\right|}^{\mathrm{CMB}}\left[\left(\mathbf{l}-\mathbf{l}_{1}\right) \cdot \mathbf{l}_{1}\right]^{2}\left[1-\left|\mathbf{l}-\mathbf{l}_{1}\right|^{2} R\left(r_{0}\right)\right] \\
+ & \frac{1}{2} \int \frac{d^{2} \mathbf{l}_{1}}{(2 \pi)^{2}} \int \frac{d^{2} \mathbf{l}_{2}}{(2 \pi)^{2}} C_{l_{1}}^{\phi}\left(r_{0}\right) C_{l_{2}}^{\phi}\left(r_{0}\right) C_{\left|\mathbf{l}-\mathbf{l}_{1}-\mathbf{l}_{2}\right|}^{\mathrm{CMB}}\left[\left(\mathbf{l}-\mathbf{l}_{1}-\mathbf{l}_{2}\right) \cdot \mathbf{l}_{1}\right]^{2}\left[\left(\mathbf{l}-\mathbf{l}_{1}-\mathbf{l}_{2}\right) \cdot \mathbf{l}_{2}\right]^{2} .
\end{aligned}
$$

This equation is equivalent to that in $\mathrm{Hu}$ (2000) for weak lensing effect on CMB anisotropy power spectrum when one drops higher order terms which are proportional to $\left[C_{l}^{\phi}\right]^{2}, R^{2}$ and $C_{l}^{\phi} R$. As we will discuss, to describe the lensing effect related to $\mathrm{CMB}$, these higher order terms are not crucial.

When one extends the perturbative calculation further, the power spectrum can be represented as a sum of a series of integrals such that

$$
\tilde{C}_{l}^{\mathrm{CMB}}=C_{l}^{\mathrm{CMB}} \sum_{i=0}^{\infty}(-1)^{i} \frac{l^{2 i} R\left(r_{0}\right)^{i}}{i !}
$$




$$
\begin{aligned}
& +\int \frac{d^{2} \mathbf{l}_{1}}{(2 \pi)^{2}} C_{l_{1}}^{\phi}\left(r_{0}\right) C_{\left|\mathbf{l}-\mathbf{l}_{1}\right|}^{\mathrm{CMB}}\left[\left(\mathbf{l}-\mathbf{l}_{1}\right) \cdot \mathbf{l}_{1}\right]^{2} \sum_{i=0}^{\infty}(-1)^{i} \frac{\left|\mathbf{l}-\mathbf{l}_{1}\right|^{2 i} R\left(r_{0}\right)^{i}}{i !} \\
& +\frac{1}{2} \int \frac{d^{2} \mathbf{l}_{1}}{(2 \pi)^{2}} \int \frac{d^{2} \mathbf{l}_{2}}{(2 \pi)^{2}} C_{l_{1}}^{\phi}\left(r_{0}\right) C_{l_{2}}^{\phi}\left(r_{0}\right) C_{\left|\mathbf{l}-\mathbf{l}_{1}-\mathbf{l}_{2}\right|}^{\mathrm{CMB}}\left[\left(\mathbf{l}-\mathbf{l}_{1}-\mathbf{l}_{2}\right) \cdot \mathbf{l}_{1}\right]^{2}\left[\left(\mathbf{l}-\mathbf{l}_{1}-\mathbf{l}_{2}\right) \cdot \mathbf{l}_{2}\right]^{2} \\
& \quad \times \sum_{i=0}^{\infty}(-1)^{i} \frac{\left|\mathbf{l}-\mathbf{l}_{1}-\mathbf{l}_{2}\right|^{2 i} R\left(r_{0}\right)^{i}}{i !}+\ldots+ \\
& +\frac{1}{n !} \int \frac{d^{2} \mathbf{l}_{1}}{(2 \pi)^{2}} \ldots \int \frac{d^{2} \mathbf{l}_{n}}{(2 \pi)^{2}} C_{l_{1}}^{\phi}\left(r_{0}\right) \ldots C_{l_{n}}^{\phi}\left(r_{0}\right) C_{\left|\mathbf{1}-\ldots-\mathbf{l}_{n}\right|}^{\mathrm{CMB}}\left[\left(\mathbf{l}-\ldots-\mathbf{l}_{n}\right) \cdot \mathbf{l}_{1}\right]^{2} \ldots\left[\left(\mathbf{l}-\ldots-\mathbf{l}_{n}\right) \cdot \mathbf{l}_{n}\right]^{2} \\
& \quad \times \sum_{i=0}^{\infty}(-1)^{i} \frac{\left|\mathbf{l}-\ldots-\mathbf{l}_{n}\right|^{2 i} R\left(r_{0}\right)^{i}}{i !},
\end{aligned}
$$

where the last term is a generalization of this series to the n-th term. The summations over $R\left(r_{0}\right)$ in each of these terms can be simplified analytically and we obtain

$$
\begin{aligned}
& \tilde{C}_{l}^{\mathrm{CMB}}=C_{l}^{\mathrm{CMB}} e^{-l^{2} R\left(r_{0}\right)} \\
& +\int \frac{d^{2} \mathbf{l}_{1}}{(2 \pi)^{2}} C_{l_{1}}^{\phi}\left(r_{0}\right) C_{\left|\mathbf{l}-\mathbf{l}_{1}\right|}^{\mathrm{CMB}}\left[\left(\mathbf{l}-\mathbf{l}_{1}\right) \cdot \mathbf{l}_{1}\right]^{2} e^{-\left|\mathbf{l}-\mathbf{l}_{1}\right|^{2} R\left(r_{0}\right)} \\
& +\frac{1}{2} \int \frac{d^{2} \mathbf{l}_{1}}{(2 \pi)^{2}} \int \frac{d^{2} \mathbf{l}_{2}}{(2 \pi)^{2}} C_{l_{1}}^{\phi}\left(r_{0}\right) C_{l_{2}}^{\phi}\left(r_{0}\right) C_{\left|\mathbf{l}-\mathbf{l}_{1}-\mathbf{l}_{2}\right|}^{\mathrm{CMB}}\left[\left(\mathbf{l}-\mathbf{l}_{1}-\mathbf{l}_{2}\right) \cdot \mathbf{l}_{1}\right]^{2}\left[\left(\mathbf{l}-\mathbf{l}_{1}-\mathbf{l}_{2}\right) \cdot \mathbf{l}_{2}\right]^{2} \\
& \quad \times e^{-\left|\mathbf{l}-\mathbf{l}_{1}-\mathbf{l}_{2}\right|^{2} R\left(r_{0}\right)}+\ldots+ \\
& +\frac{1}{n !} \int \frac{d^{2} \mathbf{l}_{1}}{(2 \pi)^{2}} \ldots \int \frac{d^{2} \mathbf{l}_{n}}{(2 \pi)^{2}} C_{l_{1}}^{\phi}\left(r_{0}\right) \ldots C_{l_{n}}^{\phi}\left(r_{0}\right) C_{\left|\mathbf{l}-\ldots-\mathbf{l}_{n}\right|}^{\mathrm{CMB}}\left[\left(\mathbf{l}-\ldots-\mathbf{l}_{n}\right) \cdot \mathbf{l}_{1}\right]^{2} \ldots\left[\left(\mathbf{l}-\ldots-\mathbf{l}_{n}\right) \cdot \mathbf{l}_{n}\right]^{2} \\
& \quad \times e^{-\left|\mathbf{l}-\ldots-\mathbf{l}_{n}\right|^{2} R\left(r_{0}\right)} .
\end{aligned}
$$

Though this expression is useful since it presents the exact calculation related to how gravitational lensing modifies the clustering power spectrum, when numerically evaluating the lensed power spectrum with a limited number of terms in this series, one cannot simply evaluate the first few terms and ignore the rest. To evaluate the contribution accurately, one should include terms which are same order in $C_{l}^{\phi}$ and $R$; For example, to the second order in $C_{l}^{\phi}$, contributions include three terms from the first line in Eq. 14, two terms from the second and a term from the third, when exponentials in each of the above lines are expanded.

Note that the lensing effect only modifies the clustering pattern of an intrinsically clustered background. If the spatial distribution of sources responsible for the diffuse background is simply Poisson, then one expects a shot-noise like angular power spectrum for fluctuations in the intensity such that $C_{l}^{\delta I}$ is a constant. In this case, Eq. 10 can be simplified and leads to $\tilde{C}_{l}=C_{l}$ such that spatial fluctuations, after lensing, remains a Poisson distribution with the same shot-noise. This is again consistent with the fact that lensing does not 
create or destroy power but only results in a modification to how power is distributed.

The same can also be deduced from Eq. 14 by taking into account the exact calculation instead of the approximate one in Eq. 10. In the case of an intrinsic Poisson-clustered power spectrum, or a smooth or slowly varying intrinsic power spectrum, one can set $C_{\left|\mathbf{1}-\ldots-\mathbf{l}_{n}\right|}^{\mathrm{CMB}}=C_{l}^{\mathrm{CMB}}$ with the substitution that $\left|\mathbf{l}-\ldots-\mathbf{l}_{n}\right| \sim l$. In this case, one can write Eq. 14 as

$$
\begin{aligned}
\tilde{C}_{l}^{\mathrm{CMB}} & =C_{l}^{\mathrm{CMB}} e^{-l^{2} R\left(r_{0}\right)}\{1+\ldots \\
& \left.+\frac{1}{n !} \int \frac{d^{2} \mathbf{l}_{1}}{(2 \pi)^{2}} \ldots \int \frac{d^{2} \mathbf{l}_{n}}{(2 \pi)^{2}} C_{l_{1}}^{\phi}\left(r_{0}\right) \ldots C_{l_{n}}^{\phi}\left(r_{0}\right)\left[\mathbf{l} \cdot \mathbf{l}_{1}\right]^{2} \ldots\left[\mathbf{l} \cdot \mathbf{l}_{n}\right]^{2}\right\},
\end{aligned}
$$

which simplifies as $\tilde{C}_{l}^{\mathrm{CMB}}=C_{l}^{\mathrm{CMB}}$ since the series within curly brackets is simply $e^{l^{2} R\left(r_{0}\right)}$. This is important since as we discuss later, lensing only modifies the clustering pattern of background sources if the distribution has distinct signatures, such that the angular power spectrum show features. In the case of a smooth distribution, which may also be Poisson, lensing does not modify the clustering pattern significantly. In this sense, most shot-noise dominated backgrounds such as radio sources and galaxy clusters through the SZ effect at arcminute scale CMB experiments, will not be modified by gravitational lensing. We find a similar situation for some what higher redshift backgrounds at IR and other wavelengths. The only lensing modification is then associated with magnification changes to source number counts (Tegmark \& Villumsen 1997) such that, down to a certain flux limit, more sources are magnified towards regions of high mass concentrations and the extent to which this is important depends strongly on the distribution of number counts as a function of flux. For truly diffuse backgrounds such as CMB and $21 \mathrm{~cm}$ emission from the general IGM prior to reionization, such an effect is not expected.

In Eq. 10 and after, $C_{l}^{\phi}\left(r_{s}\right)$ is the power spectrum of lensing potentials, for a source at a distance of $r_{s}$. In terms of the three-dimensional matter power spectrum, $P(k)$, we can write this power spectrum of lensing potentials as

$$
C_{l}^{\phi}\left(r_{s}\right)=\frac{2}{\pi} \int k^{2} d k P(k) \int_{0}^{r_{s}} d r W^{\mathrm{len}}\left(k, r_{1}\right) j_{l}\left(k r_{1}\right) \int_{0}^{r_{s}} d r_{2} W^{\mathrm{len}}\left(k, r_{2}\right) j_{l}\left(k r_{2}\right)(16)
$$

where

$$
W^{\text {len }}(k, r)=-3 \Omega_{m}\left(\frac{H_{0}}{k}\right)^{2} \frac{G(r)}{a} \frac{d_{A}\left(r_{s}-r\right)}{d_{A}(r) d_{A}\left(r_{s}\right)} .
$$




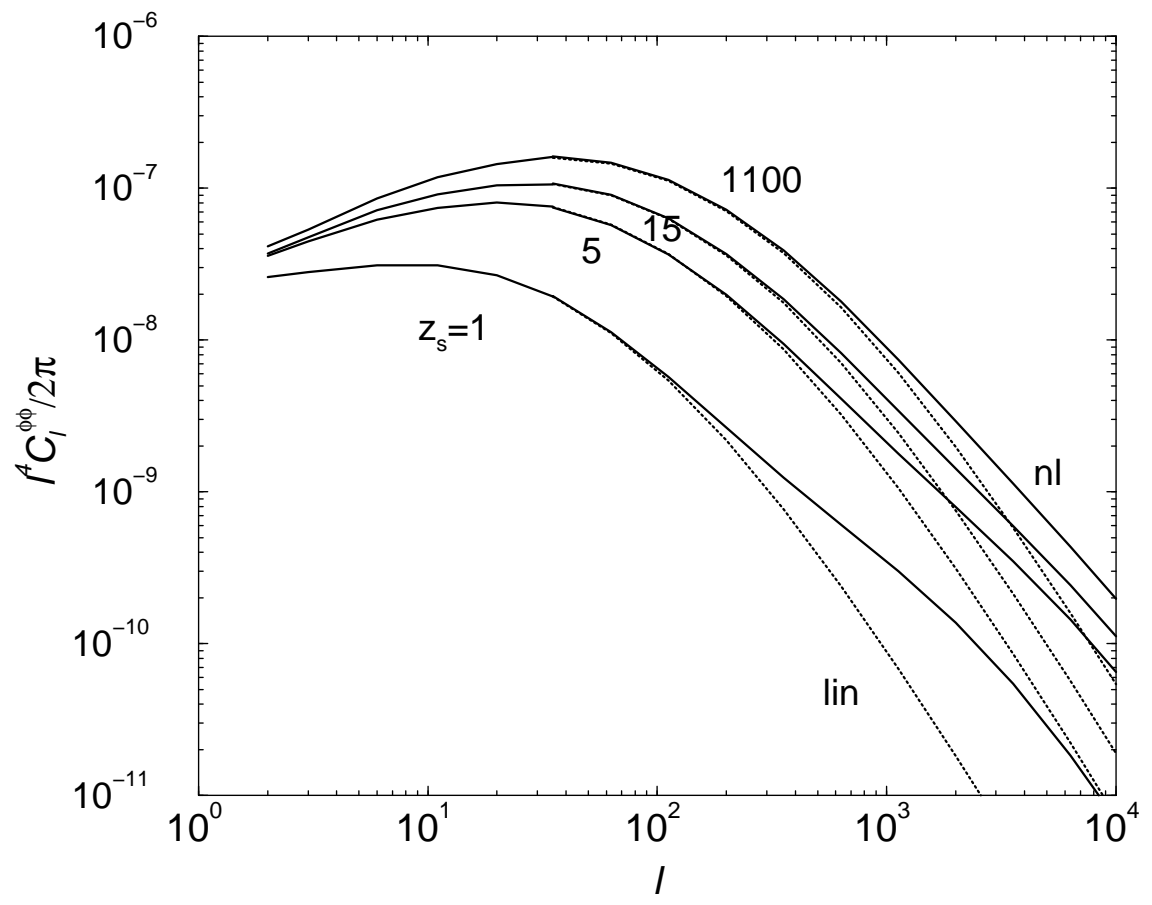

Fig. 1. Angular power spectra of the lensing deflections as a function of the source redshift $z_{s}$. The solid lines show the fully non-linear contribution to the power spectra, while the dotted lines show the contribution under linear theory power spectrum. While low redshift lensing requires a fully non-linear treatment, for sources at redshifts of 15 and higher, non-linearities do not affect lensing deflections at angular scales at least of few arcminutes and below $\left(l \sim 10^{3}\right)$.

In terms of the well known convergence, $\kappa$, power spectrum, that define the integrated surface density of mass out to the background distance $r_{s}$, one can write $C_{l}^{\kappa}=l^{4} / 4 C_{l}^{\phi}$. In calculating the matter power spectrum, we use the fitting formulae of Eisenstein \& $\mathrm{Hu}$ (1999) to evaluate the transfer function for CDM models and set the normalization to match both COBE and clusters (Bunn \& White 1999; Viana \& Liddle 1999). Note that in linear theory, the power spectrum can be scaled in time, $P(k, r)=G^{2}(r) P(k, 0)$, using the the growth function (Peebles 1980). In the non-linear regime, one can use prescriptions such as the fitting function by Peacock \& Doods (1996) or halobased approaches (Cooray \& Sheth 2002) to calculate the fully non-linear density field power spectrum.

We illustrate these power spectra as a function of $z_{s}$, the source redshift, in Fig. 1. Note that for sources at low redshifts, $z_{s} \sim 1$, non-linear corrections to the matter power spectrum is important since lensing signal from degree angular scales and below are dominated by non-linearities. As one moves to a higher source redshift, non-linear corrections move to smaller angular scales such that for sources at redshifts 15 and higher, non-linear corrections are no longer important as lensing statistics at arcminute scales are fully described by linear fluctuations in the intervening matter density field. For CMB photons, 
the rms deflection angle, $\theta_{\mathrm{rms}}=\left[\int d^{2} \mathbf{l} /(2 \pi)^{2} l^{2} C_{l}^{\phi}\right]^{0.5}$, is $2.6^{\prime}$ while for a source at a redshift of $15, \theta_{\text {rms }} \sim 2^{\prime}$. The fact that the typical deflection angle is of order few arcminutes, require that clustering modifications not be studied just to the first order perturbative correction and that higher order terms be included.

In Fig. 2, we show the CMB anisotropy power spectrum and the resulting modification related to the gravitational lensing effect. Note that the lensing effect moves power from large angular scales, where acoustic peaks are found, to small angular scales (Seljak 1996; Hu 2000). This results in an increase of power at arcminute angular scales where the damping tail of the anisotropy power spectrum is found. We also show the correction related to second order in the deflection angle power spectrum. The resulting modification related to this additional higher order correction is at the level of $20 \%$, at most, though it becomes significant as $l \rightarrow 10^{4}$, suggesting that, at small angular scales, the perturbative approach may be suspect to describe the lensing effect on CMB. Still, the approach considered in certain calculations (e.g., Hu 2000) is adequate since CMB anisotropies have no significant power at small angular scales and higher order terms, which are important at small angular scales, have a decreasing significance. For precision cosmology with arcminute angular-scale CMB power spectrum measurements, however, it may be useful to include higher order terms since they are above the cosmic variance limit of the power spectrum measurements. In addition to these higher order corrections, there are other assumptions that can complicate the interpretation of CMB lensing data. For example, the CMB power spectrum contains an anisotropy contribution from low redshifts through secondary effects such as the integrated Sachs-Wolfe effect (Sachs \& Wolfe 1967) and the SunyaevZel'dovich effect (Sunyaev \& Zel'dovich 1980). Since these contributions are also lensed, simply using lensed power spectrum with a narrow source at $r_{0}$ for cosmological parameter interpretation may be problematic. This issue is beyond the purpose of the present paper and we will leave it for a future study.

As we will now discuss, due to the smoothness of spatial fluctuations generated by low redshift diffuse backgrounds at IR wavelengths and at $21 \mathrm{~cm}$ restwavelength by neutral Hydrogen rich IGM prior to reionization, the lensing modifications, at arcminute angular scales, are not significant. This limits their ability for a lensing reconstruction in the same manner as approached for CMB.

\subsection{Diffuse Background Clustering Power Spectrum}

Since gravitational lensing deflections modify the clustering power spectrum

of anisotropies in the diffuse background, we first consider an estimate of the 
intrinsic spatial clustering signal. In the introduction, we identified several potential backgrounds that may become important for weak lensing studies including that due to first stars and galaxies in near-IR wavelengths and the $21 \mathrm{~cm}$ background by the general IGM prior to reionization. Instead of making separate estimates of their clustering signals here, we consider a general calculation under the assumption that background sources, either in the form of first stars or neutral Hydrogen, trace linear fluctuations with a bias factor that can be calculated based on the halo mass in which these sources are expected. In the case of first stars, such halos are likely to have temperatures of $200 \mathrm{~K}$, if molecular Hydrogen cooling is allowed, or $10^{4} \mathrm{~K}$, or more. In the case of diffuse emission related to, say $21 \mathrm{~cm}$, from the neutral hydrogen prior to reionization, we expect neutral Hydrogen to be present in all halos with no cut off at the low end of the mass distribution. In this case, it is likely that the neutral Hydrogen distribution is tracing the linear density field directly such that the bias factor is close to unity.

The clustering of any sources at redshifts between 10 and 30 can be calculated following standard approaches (Cooray et al. 2003). The contribution to the background intensity, say at a certain wavelength and towards a direction $\hat{\mathbf{n}}$, can be written as a product of the mean emissivity and its fluctuation

$$
I(\hat{\mathbf{n}})=\int_{0}^{\infty} d r a(r) \bar{j}(r)\left[1+\frac{\delta j(r \hat{\mathbf{n}}, r)}{\bar{j}(r)}\right]
$$

where $r$ is the comoving radial distance (or conformal time) and $\bar{j}(r)$ is the mean emissivity per comoving unit volume as a function of distance. Note that the integrand can now be identified with $\delta I(r \hat{\mathbf{n}}, r)$ in Eq. 1. In order to calculate spatial fluctuations related to the emissivity, we assume $\delta j(r \hat{\mathbf{n}}, r) / \bar{j}(r)$ trace fluctuations in the source density field, $\delta_{s}=\delta \rho_{s} / \bar{\rho}_{s}$ such that, in Fourier space,

$$
\frac{\delta j(\mathbf{k}, z)}{\bar{j}(z)}=\delta_{s}(\mathbf{k}, z) .
$$

The density field fluctuations of emitters are defined by the three dimensional power spectrum, which we define as

$$
\left\langle\delta_{s}(\mathbf{k}, z) \delta_{s}\left(\mathbf{k}^{\prime}, z\right)\right\rangle=(2 \pi)^{3} \delta_{D}\left(\mathbf{k}+\mathbf{k}^{\prime}\right) P_{s s}(k, z) .
$$

To calculate the source power spectrum, as discussed, we scale the linear clustering power spectrum by a bias factor. While in the case of $21 \mathrm{~cm}$ emission from neutral gas the source bias is expected to mostly unity, for sources that form in halos above a certain mass cut-off, $M_{\text {cut }}$, the bias factor is simply given 
by

$$
\left\langle b_{M}\right\rangle=\frac{\int_{M_{\mathrm{cut}}}^{\infty} d m m b(m) n(m)}{\int_{M_{\mathrm{cut}}}^{\infty} d m m n(m)}
$$

where $b(m)$ is the halo bias (Mo \& White 1996; Mo et al. 1997) with respect to the density field and $n(m)$ is the mass function (Press \& Schechter 1974).

Using the Limber approximation (Limber 1954), the angular power spectrum for a distribution of sources that trace a three-dimensional power spectrum $P_{s s}(k)$, when projected on the sky, is given by

$$
C_{l}=\int d r \frac{a^{2}(r)}{d_{A}^{2}} \bar{j}^{2}(z) G^{2}(r)\left\langle b_{M}(r)\right\rangle^{2} P^{\operatorname{lin}}\left(k=\frac{l}{d_{A}}, r=0\right),
$$

and the integrand is $C_{l}^{\delta I}(r)$ in Eq. 10 .

The power spectrum of diffuse emission related to sources in IR is illustrated in Fig. 3. The calculation related to $\bar{j}(z)$ in this case follows that of Cooray et al. (2003) and we make use of the most optimistic model there for illustrative purposes here. The shape of the angular power spectrum is essentially a reflection of the projected linear power spectrum over the redshift distribution of background sources. Since first stars are more likely to be found in the similar redshift range as of the neutral Hydrogen, which contributes to the $21 \mathrm{~cm}$ background, we expect the shape of the anisotropy clustering power spectrum of the $21 \mathrm{~cm}$ background to remain the same. Due to differences in emissivity, of course, the clustering power spectrum will have a different amplitude in this case.

In Fig. 3, we also show the angular power spectrum of spatial fluctuations in the background diffuse emission after gravitational lensing. While the lensing effect results in a transfer of power from arcminute angular scales, where the angular power spectrum peaks, to tens of degree angular scales, where the power spectrum is rapidly rising, there is no substantial modification to the angular power spectrum at arcminute angular scales. In comparison, as shown in Fig. 2, the lensing effect on CMB anisotropies results in a transfer of power from degree angular scales, where the power spectrum peaks in this case, to arcminute angular scales such that there is a substantial enhancement of power along the damping tail by more than two orders of magnitude. The lack of lensing modification to the clustering power spectrum of diffuse backgrounds can be understood based on Eq. 15 and results from that fact that clustering at arcminute scales is smooth and results from the collective emission from a large number of sources such that under gravitational lensing, though there may be modifications to the distribution of these sources at arcsecond angular 
scales, the distribution at arcminute angular scales remain the same. If any differences exist, these are at the level substantially below a percent. The lack of a substantial difference in the angular power spectrum before and after gravitational lensing suggests that statistics that primarily target two-point clustering information will not be useful for lensing studies. For example, the variance related to the distribution of these background fluctuations will not be substantially varying and we suggest that the variance measurements may not be ideal. The lack of a major difference also limits the extent to which lensing information can be extracted from non-Gaussian statistics, such as those related to the trispectrum that have been considered with respect lensing studies with CMB.

As discussed earlier, our calculation on the lensing effect on background clustering power spectrum may be incomplete. Since the typical deflection angle is of order an arcminute and there is substantial clustering power at arcminute scales, it is necessary that one properly accounts for higher order corrections to the previous calculations. We have attempted this numerically and show the first and second order contributions in Fig. 3. Unlike the case with CMB anisotropies, where the second order contribution was lower than the first order term, at angular scales of arcminutes and below, we find that this to be no longer the case here. In the multipole range of interest, however, we expect there to be no substantial contribution from the second and other higher order terms, though if we were to study the lensing corrections to the clustering power spectrum at arcsecond angular scales, then it may be necessary to perform an improved calculation. We restrict to arcminute scale clustering here since such clustering may easily be detectable in near future. On the other hand, even if clustering at arcsecond angular scales is detected, we expect at such angular scales, the shot-noise associated with finite density of sources that contribute to the background emission to be the dominant contribution to clustering. In Fig. 3, for illustration, we show the expected shot-noise related to the IR background generated by first stars and galaxies following calculations in Cooray et al (2003).

While we have specifically discussed the lensing modification to the IR background generated by first stars, our conclusions apply to clustering aspects of all other backgrounds. As argued earlier, for backgrounds at the same redshift range, one expects a similar shape to the angular power spectrum though the amplitude is different for obvious reasons. Our calculation related to the lensing modification of the background fluctuation power spectrum is independent of its amplitude and the difference between the lensed and unlensed power spectra scales accordingly such that the fractional difference between the two remain the same. The only differences are, however, associated with secondary considerations such as the extent to which shot-noise related to the finite density of sources that contribute to each of these backgrounds may become a source of confusion noise for lensing reconstruction. 


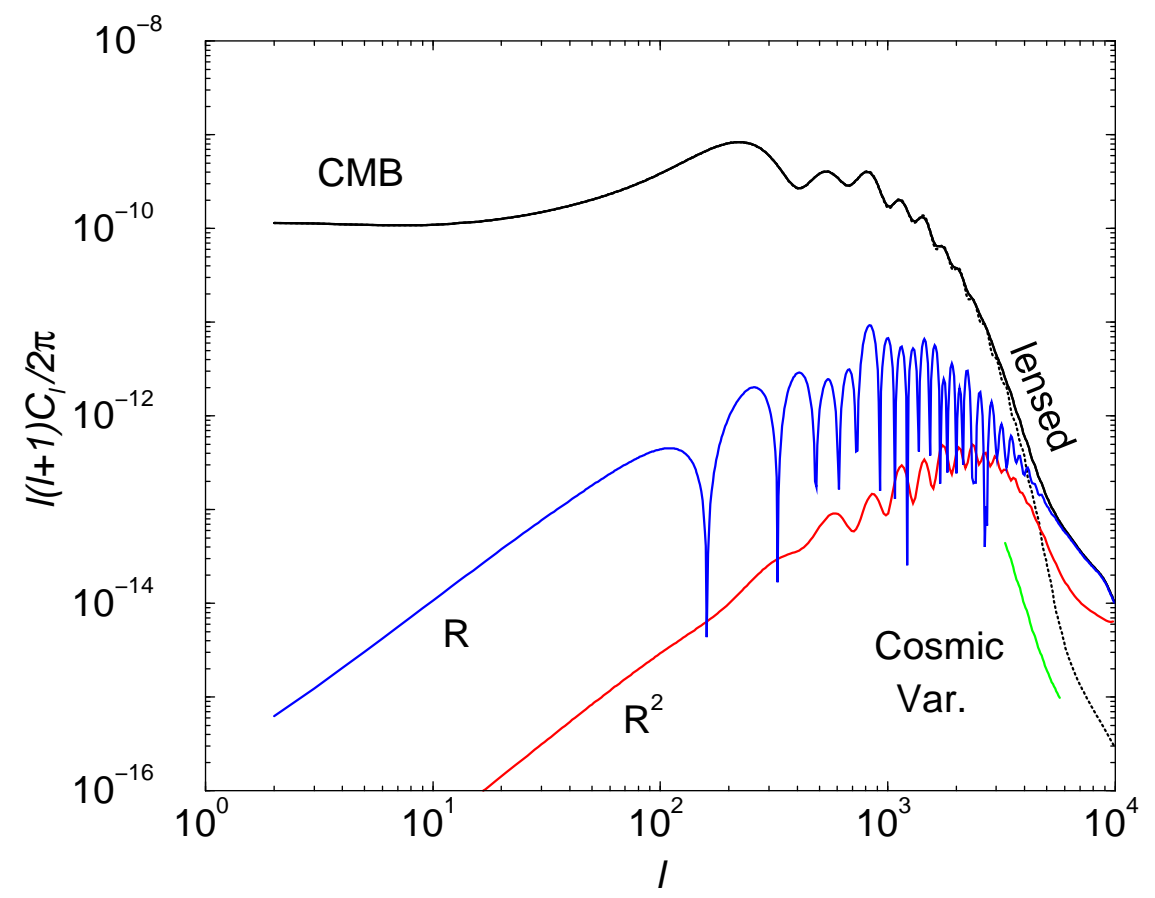

Fig. 2. The lensed CMB temperature anisotropy power spectrum. The curves labeled ' $\mathrm{R}$ ' and ' $\mathrm{R}^{2}$ ' show the lensing calculation up to the first- and second-order in $C_{l}^{\phi}$, respectively. Note that typical calculations in the literature involve the first order calculation, while the next-order correction considered here, the $\mathrm{R}^{2}$ contribution, is at the level of $30 \%$ and below and can be ignored for most practical purposes; for precision cosmology, where lensing information is used to extract cosmological parameters, such higher order corrections must be accounted for and we illustrate this why by showing the cosmic variance limit (line labled 'Cosmic Var.') of the power spectrum measurements at each of these multipoles with a full-sky experiment.

\subsection{Lensing Reconstruction from the Diffuse Emission}

As a first approach, one can consider a lensing reconstruction using diffuse background anisotropy data similar to that suggested for CMB. The idea behind here is the presence of a significant non-Gaussian signal in the form of a trispectrum, and an approach to probe this non-Gaussianity based on quadratic statistics such the power spectrum of CMB-gradients (Zaldarriaga \& Seljak 1999; Hu 2001; Hirata \& Seljak 2003). Here, we make use of the discussion in Cooray \& Kesden (2003; see, also, Hu 2001; Hu \& Okamoto 2002). Under gravitational lensing, the trispectrum for diffuse backgrounds takes the form of

$$
\begin{aligned}
& \tilde{T}^{I}\left(\mathbf{l}_{1}, \mathbf{l}_{2}, \mathbf{l}_{3}, \mathbf{l}_{4}\right)=-\int d r C_{l_{3}}^{\delta I} C_{l_{4}}^{\delta I}\left[C_{\left|\mathbf{l}_{1}+\mathbf{l}_{3}\right|}^{\phi \phi}(r)\left(\mathbf{l}_{1}+\mathbf{l}_{3}\right) \cdot \mathbf{l}_{3}\left(\mathbf{l}_{1}+\mathbf{l}_{3}\right) \cdot \mathbf{l}_{4}\right. \\
& \left.\quad+C_{\left|\mathbf{l}_{2}+\mathbf{l}_{3}\right|}^{\phi \phi}(r)\left(\mathbf{l}_{2}+\mathbf{l}_{3}\right) \cdot \mathbf{l}_{3}\left(\mathbf{l}_{2}+\mathbf{l}_{3}\right) \cdot \mathbf{l}_{4}\right]+ \text { Perm. }
\end{aligned}
$$




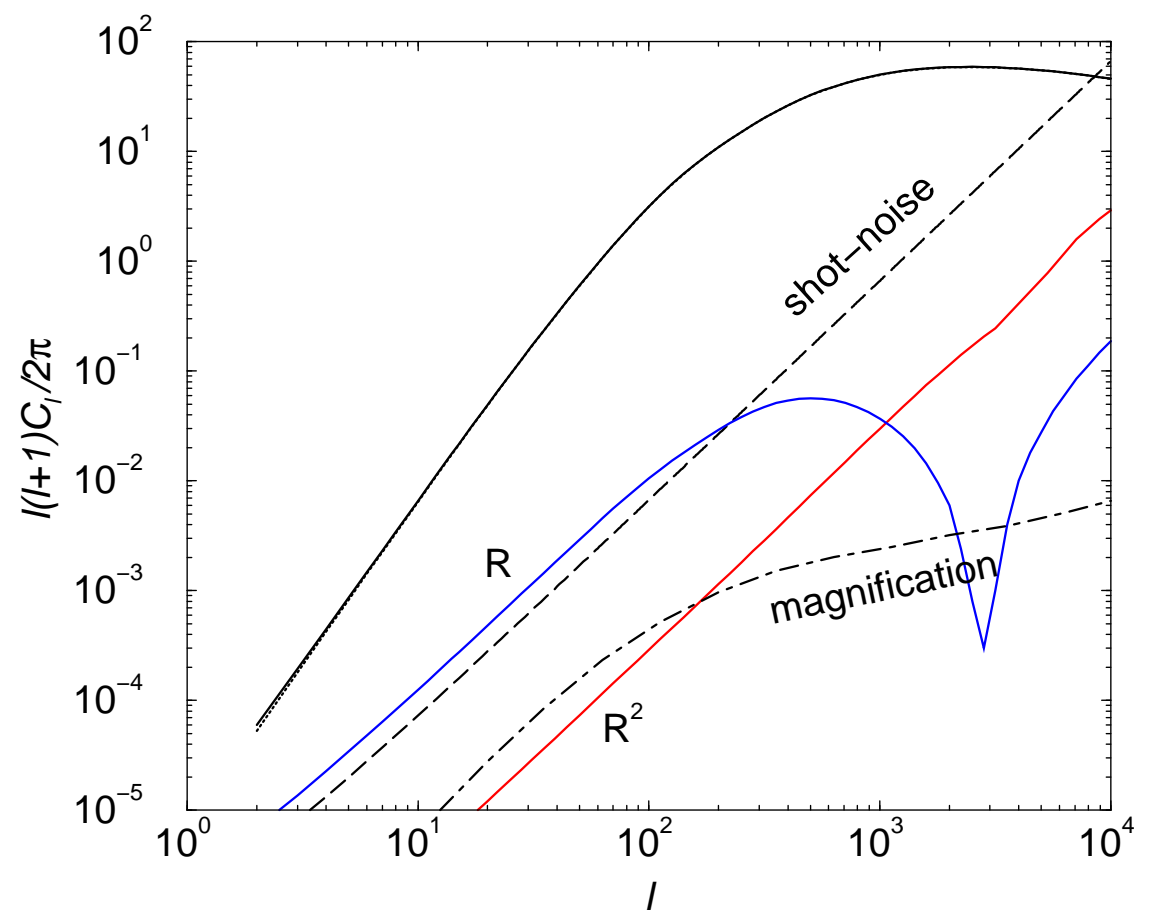

Fig. 3. The clustering power spectrum of a diffuse background at near-IR wavelengths due to first stars and galaxies and at redshifts between 10 and 30. The dotted line shows the intrinsic angular power spectrum, while the correction related to gravitational lensing is shown with a dashed line. The solid line is the angular power spectrum one observes today; at large angular scales, this power spectrum is few percent larger than the intrinsic power spectrum while the differences are insignificant at arcminute angular scales. The dashed line shows an estimate on the shot-noise contribution to the clustering signal when integrated over the finite number density of background sources; for diffuse emissions such as $21 \mathrm{~cm}$ line from the general IGM prior to reionization, we expect this shot-noise to be significantly lower or non-existent. The curves labeled ' $\mathrm{R}$ ' and ' $\mathrm{R}^{2}$ ' show the first and second-order contributions related to the lensing calculation. The curve labeled 'magnification' is an estimate on the magnification-related correction to the power spectrum when a finite number density of sources is involved and the background is studied after removing certain resolved bright sources.

where the permutations contain 5 additional terms with the replacement of $\left(l_{3}, l_{4}\right)$ pair by other combination of pairs. The derivation related to Eq. 23 can be found in Cooray \& Kesden (2003), though, we have modified it slightly to account for the finite width in the background source distribution by including an explicit integral over the radial distribution. The lensing extraction make use of this trispectrum based on the fact that it is simply proportional to $C_{l}^{\phi \phi}$.

In practice, one makes an estimator for the deflection angle based on quadratic statistics. In the present case, when compared to CMB, note that the one estimates a weighted average of the deflection angle over the redshift distribution of sources that contribute to the diffuse emission. The estimator is such that, 


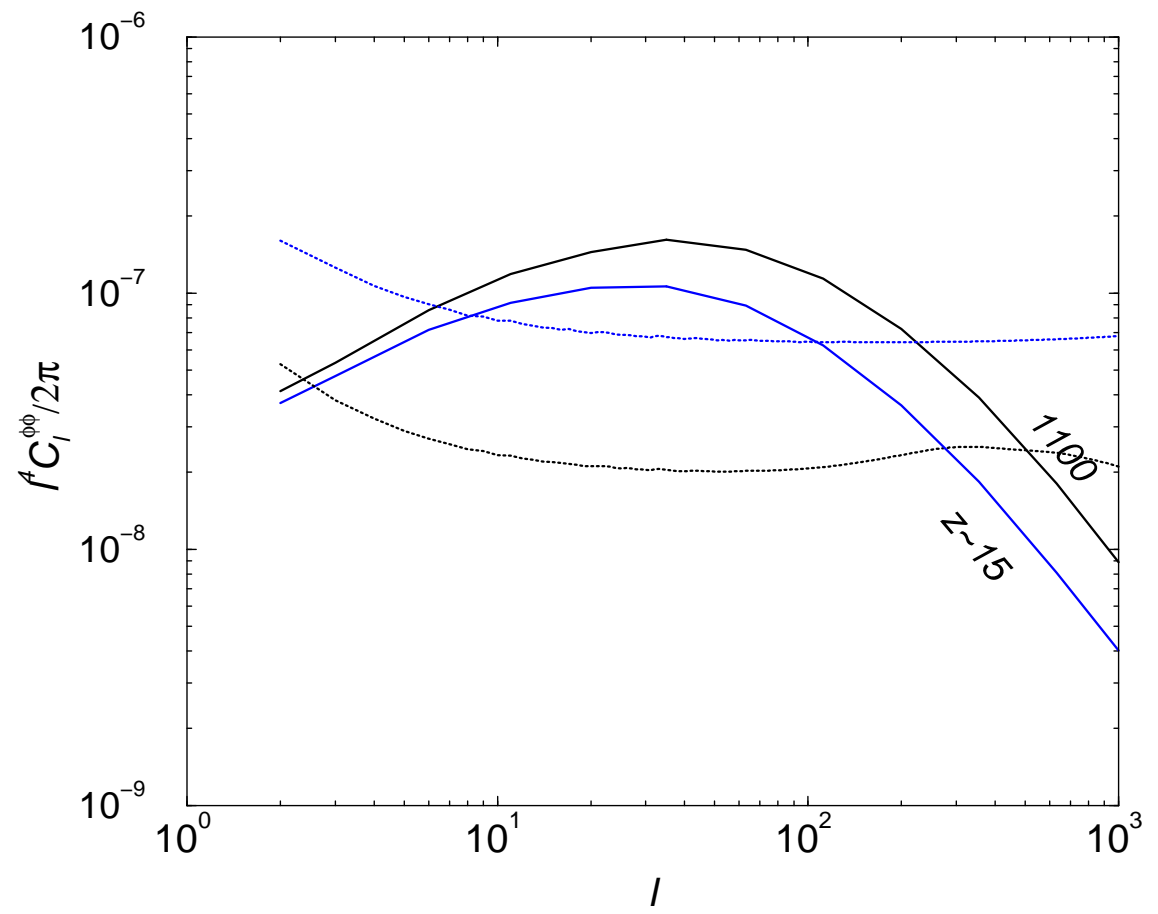

Fig. 4. The extraction of lensing information from clustering data related to the diffuse background. The solid lines show the power spectrum of projected potentials while the dotted line show the expected noise associated with the lensing reconstruction. Here, we show the case at $z \sim 15$, based on, for example, IR background from first stars and galaxies, or $21 \mathrm{~cm}$ emission from neutral Hydrogen, while, for comparison, we also show the case with CMB using, for example,e arcminute scale resolution data expected from a mission such as CMBpol. In comparison, CMB temperature fluctuations allow a significantly better reconstruction related to gravitational lensing than low redshift backgrounds and is primarily due to the difference in the fluctuation pattern on the sky or the clustering power spectrum. The presence of features and the lack of power at arcminute scales in CMB anisotropies enhance the lensing modification, while in the case of diffuse backgrounds, the lensing modifications are minor and the generated non-Gaussianities are no significant.

in Fourier space, one is convolving two filtered versions of the background intensity fluctuations

$$
\hat{I}^{2}(\mathbf{l})=\int \frac{d^{2} \mathbf{l}_{1}}{(2 \pi)^{2}} W\left(\mathbf{l}, \mathbf{l}_{1}\right) \tilde{I}(\mathbf{l}) \tilde{I}\left(\mathbf{l}-\mathbf{l}_{1}\right),
$$

where $W$ is a filter that is designed to optimally extract information related to the weak lensing effect such that the power spectrum of $\hat{I}^{2}(\mathbf{l})$ returns $\left\langle C_{l}^{\phi}\right\rangle$ where

$$
\left\langle C_{l}^{\phi}\right\rangle=\frac{\int d r C_{l}^{\phi}(r) C_{l}^{\delta I}}{\int d r C_{l}^{\delta I}}=\int d r n_{s}^{2}(r) C_{l}^{\phi}(r),
$$


where $n_{s}(r)$ is the normalized distribution of background sources that contribute to the diffuse emission. The filter, $W\left(\mathbf{l}, \mathbf{l}_{1}\right)$, can be represented by typical quadratic combinations considered in the literature, such as gradients (e.g., Zaldarriaga \& Seljak 1999). To extract all information, following the case in CMB anisotropies, the optimized filter to extract lensing information takes the form of

$$
W\left(\mathbf{l}, \mathbf{l}_{1}\right)=\frac{\left[\mathbf{l} \cdot \mathbf{l}_{1} C_{l_{1}}+\mathbf{l} \cdot\left(\mathbf{l}-\mathbf{l}_{1}\right) C_{\left|\mathbf{l}-\mathbf{l}_{1}\right|}\right]}{2 C_{l_{1}}^{\text {tot }} C_{\left|\mathbf{l}-\mathbf{l}_{1}\right|}^{\text {tot }}},
$$

such that when one constructs $N_{l}^{2}\left\langle\hat{I}^{2}(\mathbf{l}) \hat{I}^{2}\left(\mathbf{l}^{\prime}\right)\right\rangle$ one measures $\left\langle C_{l}^{\phi}\right\rangle$ with a noise contribution given by

$$
N_{l}=\left[\int \frac{d^{2} \mathbf{l}_{1}}{(2 \pi)^{2}} \frac{\left[\mathbf{l} \cdot \mathbf{l}_{1} C_{l_{1}}+\mathbf{l} \cdot\left(\mathbf{l}-\mathbf{l}_{1}\right) C_{\left|\mathbf{1}-\mathbf{l}_{1}\right|}\right]^{2}}{2 C_{l_{1}}^{\text {tot }} C_{\left|\mathbf{l}-\mathbf{l}_{1}\right|}^{\text {tot }}}\right]^{-1} .
$$

Here $C_{l}^{\text {tot }}$ is the total contribution to the angular power spectrum of the diffuse background map and includes contributions such as $C_{l}^{\text {tot }}=\tilde{C}_{l}+C_{l}^{\text {shot }}+C_{l}^{\text {mag }}+$ $C_{l}^{\text {noise }}$, which are related to the unlensed clustering power spectrum including any shot-noise, $\tilde{C}_{l}+C_{l}^{\text {shot }}$, any corrections related to the magnification, if again there is a finite number density of sources involved $C_{l}^{\text {mag }}$ and instrumental noise, $C_{l}^{\text {noise }}$.

The shot-noise associated with a finite density of sources that determine the background emission can be estimated through number counts, $d N / d S$, of the contributing sources, as a function of flux $S$, and can be written as

$$
C_{l}^{\mathrm{shot}}=\int_{0}^{S_{\mathrm{cut}}} S^{2} \frac{d N}{d S} d S
$$

where $S_{\text {cut }}$ is the flux cut off value related to the removal of resolved sources. This shot-noise acts as a source of noise for lensing reconstruction. Unlike the calculation related to the clustering spectrum, where the average emissivity is the only unknown, the shot-noise depends strongly on detailed aspects of source number counts. Since one weighs by a factor $S^{2}$, the shot-noise is more sensitive to rare objects which are brighter. Since there are many uncertainties related to establishing the source number counts precisely, we loosely estimate this following Cooray et al. (2003) and show this estimate in Fig. 3 for the case of IR-background generated by first stars and galaxies. Note that for "truly" diffuse emissions, such as the $21 \mathrm{~cm}$ rest-wavelength background from general IGM and CMB, this is not a concern.

Note that, so far, we have considered the case where the clustering analysis 
takes in to account all contributions to the background. In some situations, such as in cases where some part of the background is due to resolved sources in data, one can study clustering properties by removing such sources from the analysis. While we do not expect this to be the case either with nearIR background from first objects or in $21 \mathrm{~cm}$ emission, most dusty galaxies that contribute to the far-IR background can be resolved and removed. In such a case, the clustering power spectrum of the residual emission contain a correction associated with lensing magnification (Tegmark \& Villumsen 1997) and can be written as

$$
C_{l}^{\text {shot }}=l^{4}\left\langle C_{l}^{\phi \phi}\right\rangle S_{\text {cut }}^{4}\left[\frac{d N}{d S}\right]_{S_{\text {cut }}}^{2} .
$$

This noise contribution depends on the number counts at the flux-cut off. If this contribution can be identified, it alone may be used for a lensing study though the modification to the power spectrum is expected to be small. Though we make an estimate of the contribution in Fig. 3 (curve labeled 'magnification'), this estimate should also be considered as highly uncertain since number counts of sources that contribute to the background are not well defined in the context of backgrounds we have primarily considered. This effect is again only present in the case of a finite density of sources and is not a concern when, for example, all sources that contribute to the background have the same flux.

In addition to effects related to a finite density of sources that lead to a diffuse background, the presence of instrumental noise also affect the lensing reconstruction. This noise is given by

$$
C_{l}^{\mathrm{n}}=f_{\mathrm{sky}} w^{-1} e^{l^{2} \sigma_{b}^{2}}
$$

when $f_{\text {sky }}$ is the fraction of sky surveyed, $w^{-1}=4 \pi \sigma_{\text {pix }}^{2} / N_{\text {pix }}$ is the variance per unit are on the sky with an individual pixel noise variance of $\sigma_{\text {pix }}^{2}$ with $N_{\text {pix }}$ pixels, and $\sigma_{b}$ is the effective beam-width of the instrument.

In Fig. 4, we illustrate the extent to which $\mathrm{CMB}$ and low redshift diffuse backgrounds can be used for a lensing reconstruction by concentrating on the expected level of noise associated with the reconstructed potential power spectrum. For simplicity, here we consider arcminute scale resolution experiments, but set the noise level to be ten orders of magnitude below the clustering power spectrum such that at multipoles of few hundred, the noise-level is insignificant. This is done to consider the full extent to which diffuse backgrounds can be used for lensing studies based on clustering information and to compare with CMB related lensing studies. As shown in Fig. 4, CMB data allow an order of magnitude or better reconstruction of the projected lensing po- 
tential power spectrum when compared to the case with diffuse backgrounds. This is due reasons we have already mentioned and involve the fact that the non-Gaussianity generated in CMB data, by the nature of its fluctuation pattern, is more significant than the non-Gaussianity generated by gravitational lensing in the low redshift diffuse emission. While fluctuations in the diffuse emission may allow a reconstruction of the lensing power spectrum with a cumulative signal-to-noise ratio of order few hundred, this should be compared to at least an order of magnitude better reconstruction with CMB with the same angular resolution and fractionally the same noise level. Unlike low redshift backgrounds, CMB also has the advantage that a lensing reconstruction can be considered from polarization information in addition to temperature anisotropies we have only considered here (e.g., Guzik et al. 2001; Hu \& Okamoto 2002).

In addition to problems related to the reconstruction, there are also reasons that can complicate a simple interpretation of the reconstructed lensing power spectrum. For example, in addition to the lensing contributions we have discussed, the lensed clustering power spectrum also contains additional contributions related to the fact that the source distribution that contribute to low redshift background is broad and these lensing potentials can be correlated with the diffuse emission itself. We write the dominant contribution associated with such a cross-correlated component as

$$
C_{l}^{\mathrm{conf}}=\int \frac{d^{2} \mathbf{l}_{1}}{(2 \pi)^{2}} \int d r_{1} C_{l_{1}}^{\phi-\delta I}\left(r_{1}\right) \int d r_{2} C_{\left|\mathbf{1}-\mathbf{l}_{1}\right|}^{\phi-\delta I}\left(r_{2}\right)\left[\left(\mathbf{l}-\mathbf{l}_{1}\right) \cdot \mathbf{l}_{1}\right]^{2}
$$

This term does not exist in the case of CMB since the potentials that deflect CMB photons are disjoint in redshift space from last scattering surface where most CMB fluctuations are generated. In the case of diffuse backgrounds, if the source distribution is broad, this term can be interpreted simply as the contribution due to lensing potentials at the low end of the source distribution that is responsible for the lensing of sources at the other end of the distribution. Assuming that both potentials and the sources of background light anisotropy trace the same power spectrum $P(k)$, with a bias factor $b\left(k, r_{2}\right)$ that accounts for any departures from this assumption, this cross power spectrum can be written as

$$
C_{l}^{\phi-\delta I}\left(r_{s}\right)=\frac{2}{\pi} \int k^{2} d k P(k) \int_{0}^{r_{s}} d r W^{\mathrm{len}}\left(k, r_{1}\right) j_{l}\left(k r_{1}\right) \int_{0}^{\infty} d r_{2} b\left(k, r_{2}\right) b\left(k, r_{2}\right) j_{l}(k(2: 2))
$$

A proper accounting of effects such as this will be a necessary aspect of any study that attempt to use background source clustering for lensing purposes.

While a reconstruction with $\mathrm{CMB}$ is better, note that one reconstructs the 
potential power spectrum out to a redshift of 1100 . Thus, low redshift backgrounds are still useful for cosmological studies since they allow a reconstruction out to redshifts of 15 to 30 or so and will bridge the gap between low redshift lensing studies, based on galaxy shapes, and eventual lensing studies with higher resolution $\mathrm{CMB}$ data in the near future. In this respect, we suggest that one should concentrate further on the aspects of lensing related to diffuse backgrounds and how they can be exploited. As we have discussed, the use of non-Gaussian information for a lensing reconstruction with low redshift backgrounds, in the same manner lensing studies with CMB are proposed, is not helpful, though an attempt is still encouraged. To extract lensing information, one should concentrate on the distribution of sources, that contribute to the background emission, at arcsecond angular scales. One approach would be to use the local anisotropy of the correlation function to obtain an estimate for the shear (van Waerbeke et al. 1997); this comes from the fact while clustering properties of the background is expected to isotropic, in addition to non-Gaussianities, gravitational lensing also modify the clustering such that, locally, an anisotropic correlation function is generated. The quadrupole moment of the local correlation function captures this anisotropy and provides an estimate of shear just as ellipticity is used to estimate lensing shear. Another approach is to use variations in number counts due to lensing magnification related effects (Jain 2002), though this requires backgrounds where sources counts are easily modified by magnification due to the nature of source count distribution as a function of redshift. While we have primarily discussed the use of arcminute scale clustering information and the potential presence of non-Gaussianities for a lensing reconstruction, in a future paper, we will return to the issue of extracting lensing shear from small angular scale clustering properties.

\section{Summary}

The current weak lensing measurements of the large scale structure are mostly related to statistical study of background galaxy ellipticities. We consider a possibility to extend lensing studies with intrinsically unresolved sources and suggest that clustering properties, at arcminute angular scales, of either the point sources or the integrated diffuse emission from these point sources can be used for a lensing reconstruction. This is analogous to techniques now developed to extract lensing information from cosmic microwave background (CMB) temperature and polarization fluctuations and relies on the presence of a significant non-Gaussian signal in the background generated by the gravitational lensing modifications to the fluctuation pattern. Contrary to CMB, however, the lensing modification to the diffuse background clustering is minimal and results from the lack of distinct features in the fluctuation pattern. 
In the case of $\mathrm{CMB}$, a distinct signature or pattern exist through the acoustic nature and the lack of intrinsic power at arcminute angular scales where the damping-tail is found. We have discussed the extent to which projected matter power spectrum be reconstructed with images of the diffuse background generated by first stars and galaxies in near-IR wavelengths and the background related to $21 \mathrm{~cm}$ emission by neutral gas in the general intergalactic medium prior to reionization and have shown this to be significantly below the level of signal-to-noise ratio one can reach with, in relative terms, CMB data. This is due to the lack of significant non-Gaussianities in the diffuse emission that is generated by gravitational lensing modifications. Though limited, lensing studies with diffuse backgrounds will only be the way to extend the reconstruction of the integrated matter power spectrum out to redshifts of 15 to 30 and to bridge the gap between current and upcoming lensing studies with sources at redshifts between 1 and 2 and planned weak lensing studies with CMB out to the last scattering surface at a redshift of 1100 . As ways to improve, we suggest further studying a potential lensing reconstruction methods based on shear estimation from the local anisotropy of the correlation function of the sources that contribute to diffuse backgrounds and statistics that are optimized to extract information related to modifications associated with lensing magnification.

\section{Acknowledgments}

The author thanks Roger Blandford, Marc Kamionkowski, and Michael Kesden for useful discussions and collaborative work. This work was supported in part by DoE DE-FG03-92-ER40701 and a senior research fellowship from the Sherman Fairchild Foundation.

\section{References}

Bartelmann and P. Schneider, Phy. Rep., 340, 1 (2001).

Benabed, F. Bernardeau and L. van Waerbeke, Phys. Rev. D, 63, 043501 (2001);

Bernardeau, A\&A, 324, 15 (1997);

F. Bunn \& M. White, Astrophys. J., 480, 6 (1997).

Cooray and M. Kesden, New Astron., 8, 231 (2003)

Cooray, J. J. Bock, B. Keating, A. E. Lange and T. Matsumoto, Astrophys. J. submitted (astro-ph/0308407).

Cooray and W. Hu, Astrophys. J., 534, 533 (2000); 
Cooray and R. Sheth, Phys. Rep., 372, 1 (2002).

J. Eisenstein \& W. Hu, Astrophys. J., 511, 5 (1999).

R. Furlanetto, A. Sokasian and L. Hernquist, preprint, astro-ph/0305065 (2003).

Guzik, U. Seljak and M. Zaldarriaga, Phys. Rev. D, 62043517 (2000);

Hirata and U. Seljak, Phys. Rev. D, 67, 043001 (2003).

Hu, Phys. Rev. D, 62, 043007 (2000).

Hu, Phys. Rev. D, 64, 083005 (2001);

Hu, Astrophys. J., 557, 79 (2001);

Hu and T. Okamoto, Astrophys. J., 574, 566 (2002);

Hu \& S. Dodelson, Ann. Rev. Astro. Astrop., in press, astro-ph/0110414 (2001).

Hu and M. Tegmark, Astrophys. J. Lett. 514, 65 (1999).

T. Iliev, P. R. Shapiro, A. Ferrara and H. Martel, Astrophys. J. Lett. 572, 123 (2002).

Jain, Astrophys. J. 580, 3 (2002)

Jain and U. Seljak, Astrophys. J. 484, 560 (1997).

Kaiser, Astrophys. J., 388, 286 (1992).

Kaiser, Astrophys. J., 498, 26 (1998)

Kesden, A. Cooray and M. Kamionkowski, Phys. Rev. D 66, 083007 (2002).

Kesden, A. Cooray and M. Kamionkowski, Phys. Rev. D, 67, 123507 (2003).

Knox, A. Cooray, D. Eisenstein and Z. Haiman, Astrophys. J. 550, 7 (2001).

Limber, Astrophys. J., 119, 655 (1954).

Madau, A. Meiskin and M. J. Rees, Astrophys. J. 475, 429 (1997).

B. Metcalf and J. Silk, Astrophys. J. Lett., 489, 1 (1997).

J. Mo, Y. P. Jing and S. D. M. White, Mon. Not. Royal Astron. Soc, 284189 (1997)

J. Mo and S. D. M. White, Mon. Not. Royal Astron. Soc, 282347 (1996).

A. Peacock and S. J. Dodds MNRAS, 280 L19 (1996).

J. E. Peebles and J. T. Yu, Astrophys. J., 162, 815 (1970).

J. E. Peebles, The Large-Scale Structure of the Universe, Princeton: Princeton Univ. Press (1980).

-L. Pen, preprint, astro-ph/0305387 
H. Press and P. Schechter, Astrophys. J., 187425 (1974).

Refregier, Ann. Rev. Astron. and Astrop., 41, 645 (2003).

K. Sachs and A. M. Wolfe, Astrophys. J., 147, 73 (1967).

Scott and M. J. Rees, Mon. Not. Royal Astron. Soc, 247, 510 (1990).

Seljak, Astrophys. J., 463, 1 (1996)

Seljak \& M. Zaldarriaga, Phys. Rev. Lett., 82, 2636 (1999);

K. Sheth and B. Tormen, Mon. Not. Royal Astron. Soc, 308119 (1999).

Silk, Astrophys. J., 151, 459 (1968).

A. Sunyaev and Ya. B. Zel'dovich, Mon. Not. Royal Astron. Soc, 190, 413 (1980).

A. Sunyaev and Ya.B. Zel'dovich, Astrophys. Space Sci. 7, 3 (1970).

Tegmark and J. V. Villumsen, Mon. Not. Royal Astron. Soc, 289, 169 (1997).

Tozzi, P. Madau, A. Meiskin and M. J. Rees, Astrophys. J. 528, 597 (2000).

Van Waerbeke, Y. Mellier, P. Schneider, B. Fort and G. Mathez, Astron. \& Astrophys., 317, 303 (1997)

T. P. Viana \& A. R. Liddle, MNRAS, 303, 535 (1999).

Zaldarriaga and U. Seljak, Phys. Rev. D, 59123507 (1999);

Zaldarriaga, Phys. Rev. D, 62, 063510 (2000); 